\title{
Book Review: Teach students how to learn: strategies you can incorporate into any course to improve student metacognition, study skills, and motivation
}

Shashidhar Belbase, Hilda Horemans, Hemali Makhija, Janice Kaur Munster

Zayed University, UAE

\begin{abstract}
We who are in the profession of teaching are always concerned about student learning and performance, no matter whether it is in school or higher education. There are many factors that determine the quality of student learning, including the environment at home, in school and in the classroom; instructor and student-related factors; instructional materials; and course content. There is a lot in the literature on teaching-learning across the disciplines that looks at different approaches for classroom practices, to help students learn better and instructors to teach better. This book authored by Saundra and Stephanie McGuire (2015, Stylus Publishing) analyses four specific themes associated with teaching students how to learn: motivation, the use of Bloom's Taxonomy for student motivation, developing metacognitive skills of students, and supporting students at risk. These are the areas covered in the book's twelve chapters.
\end{abstract}

The authors first present Saundra's experience of learning how to teach, how to help students who are struggling to get good grades, developing their metacognitive skills, engaging students in deep learning, and dealing with students' psychological defense mechanisms. They then explore how to get students to take responsibility for their learning and engage students in pair or group work. The authors then try to connect brain theory to learning and explore how to get students intrinsically motivated. However, this cannot be done in isolation, so partnering with campus learning centers is crucial in helping to manage resources and time and communicating with prepared and unprepared students. They present several anecdotes of success stories; for example, at one place they state, "My students started using those strategies and began to see immediate - and in some cases remarkable - results" (p. 13). Saundra's incredible experience presented across the chapters may provide readers with a motivation to use these strategies or create their strategies to develop students' metacognitive skills.

The second theme use of Bloom's Taxonomy (BT) highlights key elements of both old and new versions of BT fitting with study cycles. The authors claim that there is a great impact of introducing BT to the classroom so that students are aware of different levels of learning from memorization and ultimately reaching to the creation of their stories. They discussed the importance of switching students from study mode to learning mode. Study mode is mechanical process aiming for higher GPA through lower level learning as per BT and learning mode is aiming for long-lasting ability to apply knowledge and skills in context, evaluate the existing knowledge and skill and create new ones that fit into the new context. It is all about making BT accessible to students and enhancing their ability to plan and implement their own metacognitive skills. The authors found BT extremely helpful in motivating students for higher order thinking, reasoning, and moving them to study for learning to teach others (real or virtual persons) as this is the best indication of content mastery. The taxonomical hierarchy of learning is not the focus of the discussion; however, it is more important in helping teachers or instructors to analyze their work or pedagogical framework. For students, it helps them analyze their learning and is an indication of which level they are in by using BT as a guideline. 
The third theme developing students' metacognitive skills extends BT further to transforming student mindset from a static/fixed one to a progressive growth mindset. This is done through a variety of activities, for example, implementing the critical reading strategy, forming collaborative groups, and putting together homework, mock exams, and classroom instructions. When students begin to evaluate their own learning together with others, they learn from each other and support each other, and this transforms their metacognitive learning skills. The authors compare how a parent's (mother's) mindset differs across cultures (in the Asian and American contexts) and how student learning is affected by it.

The fourth theme is related to supporting students at risks or low achievers by inspiring and instilling positive beliefs, helping students gain confidence in their ability to learn and perform better. This shifts their mindset i.e. that their grades do not reflect how smart they are (or not), but it reflects their attitude and effort put into the course and content materials. For this, the authors suggest different motivating techniques and how to deal with students' emotions for optimal learning. Students may go through different levels of fear and anxiety about the course, their ability to deal with personal and academic problems, and lose confidence. Such emotional disruptions may lead to their low participation and performance because they may be unaware of their potential of being successful students. The authors advocate changing teacher mindset to emphasize students' actions - moving away from the focus on grades which don't provide a complete picture of student ability.

Having had an overview of how the authors suggest this be done, how applicable is this to our context? No doubt there is a lot of value presented by the authors, given the context of her situation - students who are native speakers of English, and are highly motivated - having enrolled in the tertiary study after a rigorous admissions process. These students are likely to be more open to putting into practice the developmental strategies the author proposes, as evidenced by the case students/anecdotes presented throughout the book.

However, can the same expectations be had for the majority of students outside the context described by Saundra McGuire? Is one presentation on how metacognition works, as recommended by the authors, sufficient for students to appreciate its value? Can Bloom's Taxonomy, an understanding of which the author advocates for both lecturers and students, be properly presented given the time constraints of covering material, assessments, etc.? Furthermore, can learners incorporate these strategies independently i.e. without additional support (tutors, learning support centers, etc.)? Are all learners able to honestly evaluate their situation and identify aspects that need to be worked on, and then be able to address these satisfactorily? It is highly probable that students/learners who are outside of the North American context described by the author will have great difficulty. They will encounter many challenges being taught how to learn in the way presented in the book.

For lecturers/instructors who have been persuaded by the utility of the approach the authors advocate, the book does provide a good range of resources - samples of slides in the Appendix as well as other web-based material. These can be a springboard to the development of personalized teaching material/resources for each instructor's class/specific situation.

\section{References}

McGuire, S. Y., \& McGuire, S. (2015). Teach students how to learn: Strategies you can incorporate into any course to improve student metacognition, study skills, and motivation. Sterling, VA: Stylus Publishing, LLC. 